\title{
Leyes de cuotas electorales, Constitución y democracia
}

\author{
Cecilia Paz Valenzuela Oyaneder* \\ Alejandra Zúñiga Fajuri**
}

\begin{abstract}
RESUMEN
Se revisa el estado actual de la participación femenina en diversos ámbitos, de la mano de los más recientes estudios que ponen en evidencia la fuerza y potencia de los prejuicios que dificultan la igualdad. Se analiza, también, la doctrina sobre el ideal de la igualdad y los principios y derechos elaborados a partir de él, explicando por qué es necesaria la aplicación de medidas de compensación social y mecanismos que doten al sistema democrático de verdadera representatividad. Finalmente, se estudia el funcionamiento de los mecanismos de acción positiva así como algunas de las críticas más relevantes que se les han dirigido para los efectos de delimitar los posibles alcances de las medidas de cuotas electorales aplicables en Chile.
\end{abstract}

Democracia - igualdad - cuotas electorales de género

\section{Gender quota Law, Constitution and Democracy}

\begin{abstract}
This paper reviews the current status of women's political participation with the most recent studies that demonstrate the power and strength of the prejudices that binder equality. We analyze the doctrine of equality rights explaining why it is necessary to apply social compensation measures, and mechanisms to ensure true representation. Finally, we study the performance of affirmative action mechanisms and some of the most relevant criticism with the purpose of delimiting the possible scope of the egalitarian measures suitable in Chile.
\end{abstract}

Democracy - equality - gender quotes

* Magíster en Derecho Constitucional, Universidad de Valparaíso. Chile. Abogada, Universidad de Playa Ancha de Ciencias de la Educación. E-mail: valenzuelaoyaneder@gmail.com

** Doctora en Derecho, Universidad Autónoma de Madrid. Escuela de Derecho de la Universidad de Valparaíso. Chile. Escuela de Derecho de la Universidad Diego Portales, Santiago, Chile. Errázuriz 2120. Valparaíso.32-250 7014/32-2507180. alejandra.zuniga@uv.cl

Artículo recibido el 15 de enero de 2014 y aceptado para su publicación por el Comité Editorial el 28 de mayo de 2014. 


\section{INTRODUCCIÓN}

$\mathrm{E}$ 1 pasado 23 de abril la presidenta Bachelet firmó el proyecto de ley que propone cambios al sistema electoral binominal -hacia un sistema proporcional- para aumentar el número de escaños en el Congreso (de 120 a 154 el de diputados y de 38 a 50 el de senadores) y, además, incorporar una ley de cuotas que impida que algún partido pueda presentar más del $60 \%$ de candidatos del mismo sexo, asegurando al menos el $40 \%$ de candidatas femeninas. Este proyecto podría permitir en la eventualidad subir del actual $17 \%$ de participación en el Congreso y superar la media regional que se encuentra en $23 \%$.

La pretensión de autorizar medidas de discriminación positiva en Chile no es nueva. Ya en la década del 80 se comenzó a cuestionar el complejo escenario que presenta una ciudadanía femenina que se reconoce incompleta, pues, aunque la mujer lleva casi tres décadas votando, su participación en el ámbito público es extraordinariamente escasa. A nivel mundial, según datos aportados por la Unión Interparlamentaria, su inclusión en los parlamentos no sobrepasa el 20\%, no obstante representar el $50 \%$ del electorado.

Esta realidad, que se origina por un contexto sociocultural que obstruye la inclusión de las mujeres en política imponiéndoles obligaciones familiares y domésticas, se genera también por factores netamente políticos como el "impacto negativo del sistema electoral binominal en la inserción de las mujeres en la política, el funcionamiento cerrado y tradicional de los partidos políticos y la ausencia de medidas de discriminación afirmativas que permitan incrementar el acceso de las mujeres a espacios de poder político, particularmente en el Parlamento" (CIDH, 2009:112). Cobran relevancia, entonces, los acuerdos alcanzados en la Plataforma para la Acción de Beijing 95, en los que se planteó la necesidad de adoptar una particular medida de acción afirmativa: la ley de cuotas electorales, que hasta ahora ha permitido un incremento significativo en "la presencia de mujeres en las legislaturas en un promedio de nueve puntos de porcentaje, demostrando su efectividad para aumentar la representación política de la mujer en la región” (Buvinic y Rosas, 2004:5).

Aún más, el mecanismo de las cuotas de género ha trascendido la esfera pública en el caso de algunos países europeos como Francia, Holanda, Italia y Bélgica, que a principios del 2011 aprobaron leyes que imponen una cuota mínima de mujeres en órganos de decisión de sus empresas, porque ellas ocupan solo el 12\% de los puestos de los consejos de administración en el sector privado. Un porcentaje menudo si se tiene en cuenta que, desde hace años, las mujeres suponen el $60 \%$ de los graduados universitarios (Sauquillo, 2008). Ahora, si bien las cuotas se perfilan como medidas funcionales para subvertir la baja participación femenina en distintos ámbitos de la sociedad y, especialmente, en la política, hay quienes todavía cuestionan su sostén jurídico o su legitimidad moral. ¿Se puede responder a las críticas que se les han dirigido en relación con el principio de igualdad y la integridad del proceso democrático? Este trabajo intentará demostrar, no solo su compatibilidad y justificación basada precisamente en el ideal de la igualdad, sino también su legitimidad moral y pertinencia en términos de una verdadera democracia "representativa". 
Para ello, en primer lugar, vamos a revisar el estado actual de la participación femenina en diversos ámbitos, de la mano de los más recientes estudios que ponen en evidencia la fuerza y potencia de los prejuicios que dificultan la igualdad. A continuación se pasa revista brevemente a la doctrina que, a partir del análisis del ideal de la igualdad y los principios y derechos elaborados a partir de él, explica por qué es necesaria la aplicación de medidas de compensación social, en los términos referidos por Rawls en su Teoría de la Justicia. También se destaca que las cuotas son exigidas en función de argumentos asociados a la naturaleza y calidad de la representación democrática. Finalmente, se estudia el funcionamiento de los mecanismos de acción positiva y algunas de las críticas más relevantes que se les han dirigido para los efectos de delimitar los posibles alcances de las medidas de cuotas electorales aplicables en Chile.

\section{Por Qué SON NECESARIAS las MEdidas de ACCión POSITIVA:}

\section{ALGUNOS DATOS RELEVANTES}

Si asistiéramos a las clases de ciencia de la Universidad de Yale probablemente no apreciaríamos disparidades de género, pues en las clases y conferencias parece haber participación de un número igual de estudiantes varones y mujeres. Se suele suponer que los científicos, que normalmente se jactan de pensar objetivamente, son totalmente inmunes a la discriminación de género. Sin embargo, un reciente estudio realizado por Corinne Moss-Racusin y sus colegas de la Universidad de Yale sugieren lo contrario, que a pesar de los esfuerzos para reclutar y retener a más mujeres, una patente disparidad de género persiste dentro de la ciencia académica. La pregunta relativa a las razones de las disparidades de género en el área de las ciencias, la tecnología, ingeniería y matemáticas se suele responder afirmando que las mujeres son biológicamente menos capaces que los hombres en los campos cuantitativos o que ellas optan por evitar un estilo de vida propio de un científico. Siempre se asume que los prejuicios de género no existen en ciencias y es por ello que el resultado y las implicaciones de este estudio son alarmantes, no solo para las mujeres que buscan un cargo profesional en el área de las ciencias duras, sino también para todo el campo de la ciencia en general.

Si bien muchas investigaciones habían demostrado el sesgo de género en distintos grupos demográficos, faltaba investigar experimentalmente si las facultades de ciencias muestran un sesgo en contra de las estudiantes, que pudiese contribuir a la disparidad de género. En el estudio de Moss-Racusin, aleatorizado y doble ciego $(n=127)$, se construyeron 127 postulaciones idénticas para perfilar a un candidato que buscaba empleo y tutoría en seis facultades universitarias de biología, química y física en Estados Unidos. Se pedía precisar el empleo ofrecido, condiciones del puesto y salario anual. Las 127 solicitudes eran iguales en todo, excepto en el nombre del candidato: en unas era Jennifer y en otras, John.

El resultado fue revelador. A pesar de ser una encuesta inventada, con un candidato inventado, los "John" no solo obtuvieron más ofertas de trabajo que las "Jennifer", sino que también más dinero: 4.000 dólares anuales más de sueldo. Asimismo, las 
condiciones de trabajo fueron mejores para ellos que para ellas, incluyendo días de vacaciones y permisos. En algunos casos, la oferta salarial para Jennifer alcanzó solo al $75 \%$ del sueldo ofrecido a John (en Ciencias Matemáticas); en otros, al 84\%, como en psicología (Moss-Racusin, 2012:164-74).

\section{Cuadro 1}

Comparación del salario de mujeres y hombres en ciencias

\begin{tabular}{lccc}
\hline Ocupación & $\begin{array}{c}\text { Salario femenino } \\
\text { como porcentaje } \\
\text { del masculino }\end{array}$ & $\begin{array}{c}\text { Salario } \\
\text { femenino } \\
\text { medio }\end{array}$ & $\begin{array}{c}\text { Salario } \\
\text { masculino } \\
\text { medio }\end{array}$ \\
\hline Ciencias Matemáticas & $75,7 \%$ & $\$ 56.000$ & $\$ 74.000$ \\
\hline Biología / Ciencias de la vida & $85,2 \%$ & $\$ 52.000$ & $\$ 61.000$ \\
\hline Computación y ciencias informáticas & $88,8 \%$ & $\$ 71.000$ & $\$ 80.000$ \\
\hline Ciencias físicas & $76,9 \%$ & $\$ 50.000$ & $\$ 65.000$ \\
\hline Ciencias sociales & $90,0 \%$ & $\$ 63.000$ & $\$ 70.000$ \\
\hline Psicología & $84,6 \%$ & $\$ 55.000$ & $\$ 65.000$ \\
\hline
\end{tabular}

\section{Cuadro 2}

Resultados del estudio en relación con el salario ofrecido al estudiante hombre y mujer ${ }^{1}$

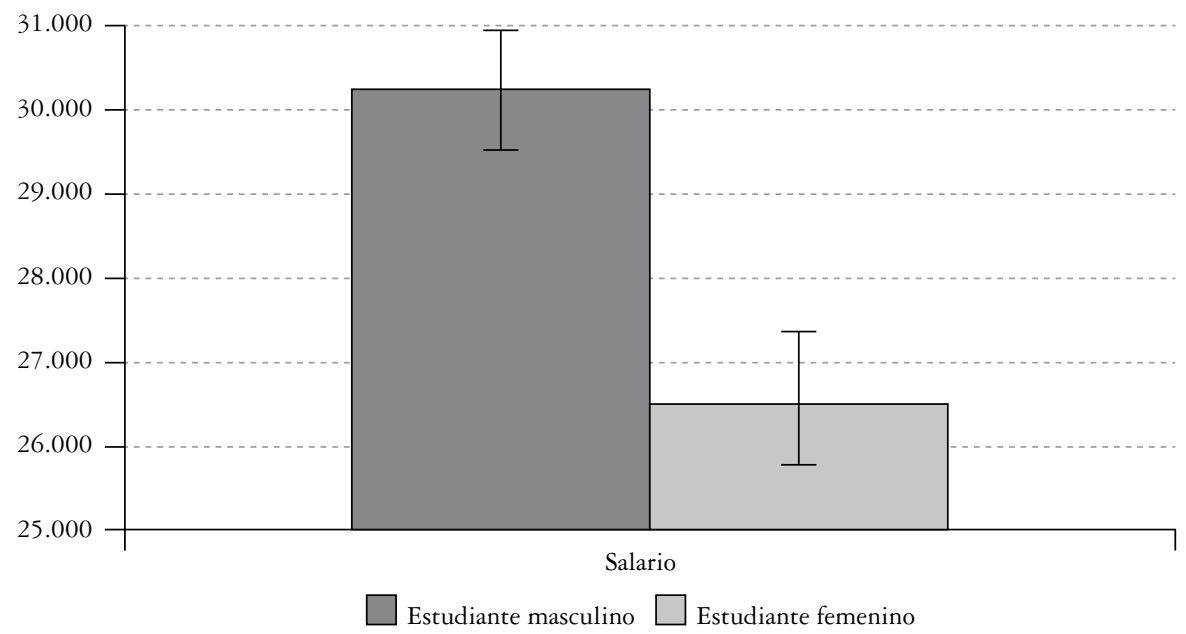

${ }^{1}$ Ambos cuadros en el estudio de Moss-Racusin (2012). 
La conclusión del estudio, entonces, fue que "los participantes de las facultades clasificaron al solicitante varón como significativamente más competentes y contratables que el solicitante (idéntico) mujer. Los participantes también seleccionaron un salario inicial más alto para los varones y les ofrecieron más orientación a su carrera. El sexo de los participantes académicos no afectó las respuestas, de modo que todos eran igualmente propensos a presentar un sesgo negativo en contra de las estudiantes... Estos resultados sugieren que las intervenciones que abordan los prejuicios de género en los profesores podrían avanzar hacia el objetivo de incrementar la participación de las mujeres en la ciencia" $^{2}$. Pero veamos otro caso.

Como se sabe, el espíritu empresarial es un camino fundamental para la creación de empleo, el crecimiento económico y la prosperidad. En las primeras etapas de la creación de empresas (star up), la presentación de los proyectos empresariales a los inversores es críticamente importante. Generalmente se piensa que la propuesta de negocio y la experiencia son los principales criterios para las decisiones de inversión. Sin embargo, la reciente investigación de la doctora del MIT Fiona Murray, coautora del artículo "Investors prefer entrepreneurial ventures pitched by attractive men", concluye otra cosa. La investigación, realizada por académicos de la escuela de negocios de Harvard, Wharton y del MIT, intentó medir el efecto que tenía el género y el aspecto físico a la hora de recaudar fondos de riesgo.

El estudio está basado en dos experimentos: uno con presentaciones orales de planes de negocios de star ups reales, y otras en video. En el primer estudio, en el que participaron 521 personas, se eligió en $68 \%$ de las ocasiones las presentaciones narradas por hombres. En el segundo, otras 207 personas contemplaron videos donde se mostraban los planes de negocio. Se les preguntaron qué probabilidad de invertir en la star up tenían y se les pidió que evaluaran el atractivo de los ejecutivos. La conclusión fue que los hombres emprendedores tenían $60 \%$ más de probabilidad que las mujeres para lograr el éxito al buscar financiación. Además, el estudio encuentra que el género explica el $42 \%$ de esta variación.

Así, los inversores prefieren propuestas de negocios presentadas por varones, aun cuando el contenido de la propuesta sea el mismo. El estudio presentó los mismos planes de negocios, primero, usando una voz masculina, y luego, la de una mujer. A continuación se les pidió que evaluaran las ideas, y los resultados fueron contundentes. Ante los mismos planes de negocios, los inversionistas preferían aquellas ideas expuestas por hombres $68 \%$ de las veces. Este efecto era moderado por el atractivo físico masculino: el varón atractivo resulta particularmente persuasivo, mientras que el atractivo físico no importó ventaja alguna entre las mujeres empresarias (Wood et al., 2014).

\footnotetext{
${ }^{2}$ Otro estudio que ha demostrado similares resultados es el llamado "Howard and Heidi", de Anderson y Flynn, citado en Toegel and Barsoux."Women Leaders: The Gender Trap”, The European Business Review, Número de julio de 2012.
} 


\section{ACCIÓN POSITIVA Y PRINCIPIO DE IGUALDAD}

\subsection{Las cuotas como mecanismos de compensación social}

\section{Gráfico 1}

Diputados(as) elegidos(as) entre 1989-2009

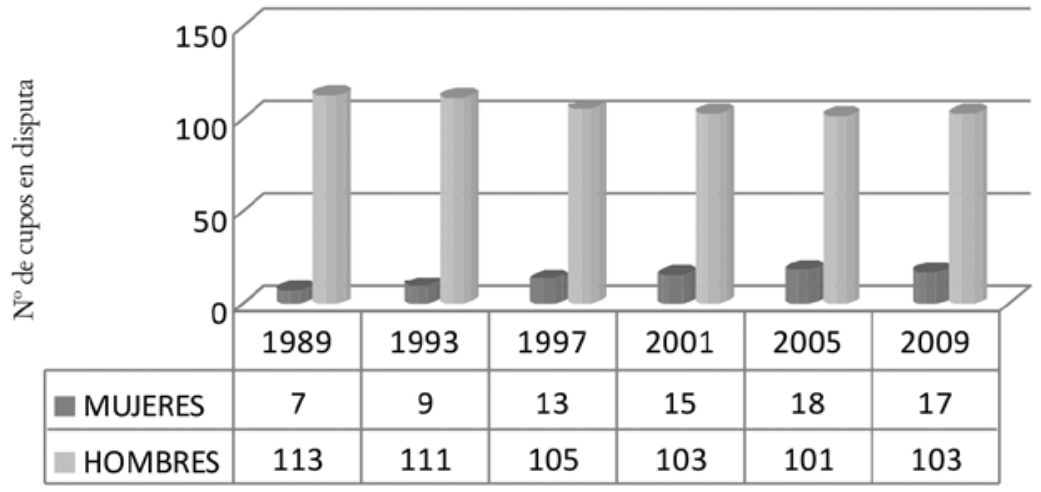

Gráfico 2

Situación de la participación femenina en el Congreso Nacional desde 1990 al 2011

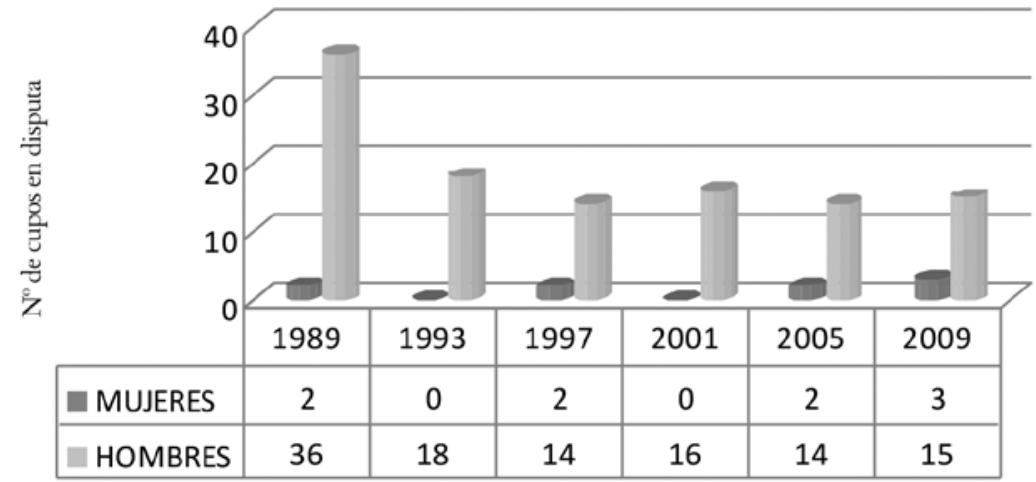

Fuente: Datos oficiales Servel.cl

El principio de igualdad, en la teoría de Rawls, se sustenta en el reconocimiento de que el ideal de la meritocracia es un mito. Por ello, las políticas de compensación están destinadas a garantizar la igualdad efectiva de oportunidades. Ellas abogan por la inclusión de instrumentos capaces de "emparejar la cancha”, es decir, de superar la 
discriminación subyacente que ciertos grupos sociales sufren desde hace mucho y que no les permitirán nunca, sin que medien estos mecanismos compensatorios, competir en igualdad de condiciones.

Dicho de otro modo, la justificación moral de las medidas de discriminación positiva descansa en la injusticia de la discriminación precedente que ellas tratan de eliminar, obligando a los más aventajados a compartir sus privilegios y los espacios de poder que, inmerecidamente (es decir, solo en razón de su pertenencia a un determinado grupo), han mantenido a lo largo del tiempo. Posiblemente el Cuadro siguiente ayude a graficar el funcionamiento del segundo principio de justicia de Rawls.

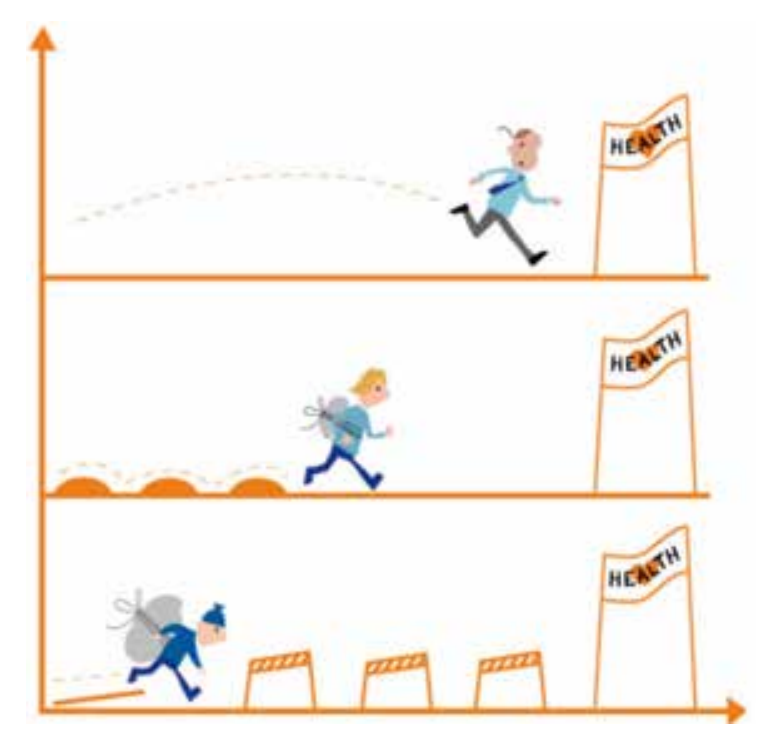

Fuente: "Norwegian Ministry of Health and Care Services". National strategy to reduce social inequalities in bealth. Report No 20, Noruega, 2006-2007.

Como permite apreciar el Cuadro, se trata de una carrera en la que a algunos corredores se les ha asignado una pesada carga porque pertenecen a un determinado grupo social. A causa de esta desventaja, el corredor medio con carga quedará rezagado del corredor medio sin carga, aunque algunos corredores con carga adelantarán a algunos corredores sin carga. Imaginemos ahora que alguien agita una varita mágica y las cargas desaparecen de la espalda de todos los corredores. Si los dos grupos de corredores son iguales en capacidad, la distancia entre aquellos con carga y sin carga dejará de aumentar, aun cuando aquellos que sufrían por la discriminación previa no logren nunca equipararse. Más aún, si se tratara de una carrera donde los padres que van primero pudieran pasar el relevo a sus hijos, no habría igualación de la carrera ni siquiera por medio de generaciones. La carrera solo podría volverse limpia si cada uno es obligado a parar y comenzar de nuevo en la misma línea de salida, y si todos los que no llevaban carga 
fueran obligados a cargar peso hasta que las diferencias en el promedio de ejecución de los grupos desaparecieran o, alternativamente, si quienes han sufrido desventajas en el pasado recibieran privilegios especiales hasta que se equiparasen ${ }^{3}$.

Este Cuadro nos faculta para precisar el mandato específico que se deriva del principio de igualdad y que se relaciona con sus dos subprincipios: la igualdad por equiparación y la igualdad por diferenciación (Alexy, 2008:351). La igualdad por equiparación, en tanto manifestación de la igualdad ante la ley, supone un trato igual de circunstancias o de situaciones no idénticas que, sin embargo, se estima deben considerarse irrelevantes para el disfrute o ejercicio de determinados derechos o para la aplicación de una misma reglamentación. La igualdad por diferenciación consiste en una desigualdad en el trato basada en la existencia de condiciones relevantes respecto de los efectos de las normas (Peces Barba, 1996:7).

En el primer caso se incluye la norma que considera a características como la raza o el sexo como irrelevantes para los efectos de reconocer el derecho a voto. En el segundo caso esas mismas características resultarían relevantes para los efectos de autorizar, por ejemplo, una política de discriminación positiva para el acceso igualitario a la universidad o a cargos de elección popular. Por cierto que dicha diferenciación no debe carecer de justificación que la legitime, pues siempre ha de probarse la necesidad del trato desigual, ya que es el trato igualitario la regla general (Alexy, 2008:360).

Para la aplicación de un trato diferenciado será imprescindible contar con juicios que determinen que ciertos rasgos o circunstancias fácticas son relevantes, los cuales constituirán finalmente su razón justificatoria, pues -recuerda Irving (2008:181) - la igualdad racial, las leyes afirmativas o los programas diseñados para lograr la igualdad de género, o para mitigar las desventajas basadas en el género, no son derogaciones del principio de igualdad, sino que ellas son la forma de darle efecto.

\subsection{Cuotas y representación democrática}

Las leyes de cuotas electorales son deseables y justas no solo como mecanismo de compensación por la discriminación estructural. También es una demanda por una "democracia paritaria" que, en el entender de Yanira Zúñiga, más que una propuesta de participación equilibrada de mujeres y hombres en los procesos decisorios políticos, se trata de un reclamo de orden social en un cuadro de responsabilidades compartidas tanto en el ámbito público como en el privado-doméstico. En esa línea, se trata de un concepto de "sociedad integrada a partes iguales por mujeres y por hombres, en la que la representación equilibrada de ambos en las funciones decisorias de la política es condición previa al disfrute pleno y en pie de igualdad de la ciudadanía, y en la cual unas tasas de participación similares o equivalentes (entre el 40 y el 60\%) de mujeres

3 "Norwegian Ministry of Health and Care Services". National strategy to reduce social inequalities in health. Report No. 20, Noruega, 2006-2007. 
y hombres en el conjunto del proceso democrático es un principio de democracia" (Zúñiga, 2005:131-154).

Para Leuchprecht, la democracia partidaria es un concepto que reconoce el hecho de que la democracia solo puede ser auténtica cuando "abraza a los seres humanos tal como realmente son, mujeres y hombres, y no como algunas formas de entidades abstractas y neutrales. Implica que ambos, hombres y mujeres, deberían participar en los procesos de adopción de decisiones sobre una base de paridad en torno al 50\%, y que tendrá que existir una auténtica distribución de las responsabilidades familiares entre ambos sexos. Va más allá de la distribución entre vida privada y pública: reconoce que la vida pública es privada" (Leuchprecht, 1996). La paridad democrática, entonces, se entiende en torno a lo que se conoce como representación descriptiva, sustantiva y también, para algunos, simbólica.

Para los teóricos políticos la representación descriptiva requiere la presencia de miembros de un determinado grupo social en el ente legislativo, la que mejora cuando la proporción en el Congreso se acerca a la proporción real de ese grupo (las mujeres, por ejemplo) en la población (cercana al $50 \%$ ). La representación sustantiva, a su turno, se refiere a la promoción de los intereses de cierto grupo en el proceso legislativo y esta mejora cuando una mayor cantidad de temas importantes para el grupo de "las mujeres" ingresan a la agenda parlamentaria y son eventualmente incorporados en la legislación. Finalmente, la representación simbólica alude a cómo los representantes "son percibidos y evaluados por aquellos que representan" y esta representación progresa cuando la población como un todo comienza a tener actitudes más igualitarias respecto de las capacidades de las mujeres como funcionarias elegidas (Franceschet, 2008: 61-2). Muchos defensores de las cuotas de género afirman que la mayor presencia de mujeres conducirá a una mayor atención respecto de sus prioridades políticas. Luego, aun cuando las cuotas de género están más llanamente dirigidas a mejorar la representación descriptiva, la mayoría de los argumentos termina apelando a razones de representación sustantiva o simbólica.

Lo cierto es que existe considerable evidencia empírica respecto de la mayor probabilidad de que las legisladoras promuevan los intereses de las mujeres que sus contrapartes masculinos. Además, la presencia de grupos históricamente marginados en cargos de elección popular desafía los estereotipos arraigados respecto de su capacidad para ostentar cargos políticos (Mansbridge, 1999). Ahora bien, si bien el vínculo entre representación descriptiva y simbólica no genera gran controversia (pues la mayoría considera que tener mayor cantidad de mujeres en política permite derrumbar estereotipos negativos sobre su capacidad), el vínculo entre representación descriptiva y sustantiva sí incomoda, en especial, debido a la ambigüedad de la idea de "intereses de las mujeres". La heterogeneidad de las mujeres como grupo hace problemático asumir que tengan un conjunto compartido de intereses. Más aún, el asumir que las mujeres como grupo tienen un conjunto de intereses políticos compartidos reforzaría ideas esencialistas falsas, pues las experiencias de género están matizadas por otros aspectos de su identidad, como el origen étnico, la clase social, la religión, etcétera.

A esta crítica se responde sosteniendo que la presencia de mujeres en entidades legislativas es importante precisamente debido a la ambigüedad de sus intereses, pues si 
ellos fueran claros y obvios, entonces teóricamente cualquiera podría representarlos. "Por eso es importante tener miembros de ese grupo representados en el cuerpo legislativo en una proporción más o menos aproximada a su presencia en la población. Las experiencias individuales de representantes descriptivamente similares a los miembros de un cierto grupo históricamente marginado informarán respecto de las perspectivas que llevan consigo a su cargo, aumentando la probabilidad de que tengan una lectura más precisa de los intereses de un grupo en particular, aun cuando estos no sean inmediatamente claros" (Franceschet, 2008:65). En definitiva, señala Anne Phillips, el argumento del interés no depende de establecer un interés unificado para todas las mujeres: más bien depende de establecer una diferencia entre los intereses de las mujeres y los intereses de los hombres $(1995,68)$.

Por cierto, la amplia mayoría de estudios en Norteamérica y Europa apoya la aseveración de que tener una mayor cantidad de mujeres en cargos públicos hace una importante diferencia, pues es más probable que las legisladoras (y no los legisladores) le den prioridad a los temas de género y patrocinen una legislación promujer (Franceschet, 2008:69-70). Es por ello, sostiene Marcela Ríos (2006:30), que la necesidad de las cuotas de género electorales se fundamenta, primero, en el argumento de funcionamiento de la democracia, pues el derecho de todo ciudadano a elegir a sus representantes y a ser elegido como tal no se garantiza igualitariamente a las mujeres. En segundo lugar, se funda en la justicia social, pues si las mujeres representan más de la mitad de la población mundial, debieran también tener la mitad de los puestos de representatividad. Tercero, se defiende en atención al argumento de las experiencias, pues las vivencias de las mujeres -sociales, biológicas o socialmente construidas- deben ser representadas en el Parlamento. Y cuarto, se apela al argumento de defensa de los intereses de grupos específicos, porque mujeres y hombres pueden tener intereses distintos, es necesaria la presencia de ellas en los órganos democráticos para que se vean representados.

\section{Acciones POSITIVAS: CRÍticas y DEFEnSA}

\subsection{Acciones positivas y cuotas de género}

Estados Unidos fue pionero en aplicar medidas de discriminación inversa en materia de educación y de empleo. Conocido es el caso Regents of the University of California versus Bakke, de 1978, en el que la Corte Suprema resolvió la situación de un estudiante blanco, Allan Bakke, que demandó a la Universidad de California en Davis por no haber podido ingresar a su Facultad de Medicina en aplicación de la reserva de 16 de las 100 plazas para nuevo ingreso a determinadas minorías raciales, en circunstancias que por su calificación académica hubiese podido ingresar sin problemas. En su veredicto, la Corte falló a favor de Bakke a base del Acta de Derechos Civiles que prohíbe la exclusión de cualquier persona en actividades que reciban aporte financiero federal por motivos de raza, color o nacionalidad. Ronald Dworkin rechazó la sentencia sobre la base de que la cláusula de igual protección garantiza el tratamiento como igual -con 
igual consideración y respeto- en el proceso político y las deliberaciones que implican decisiones como adoptar una medida que vaya en detrimento de otros, solo mientras ello está de acuerdo con el interés general. Es decir, si bien Allan Bakke no era responsable de la necesidad de implementar medidas tendientes a mejorar la integración racial en los EE.UU., no estaba eximido de que esas decisiones le afectaran, ya que no es objeto de la cláusula de igual protección proteger a los ciudadanos de todas las distinciones o clasificaciones legales que juegan en su contra, sino garantizar el tratamiento de las personas “con igual consideración y respeto" (Dworkin, 2003:451).

En la misma década se conoció el caso DeFunis v. Odegaard en el que un estudiante blanco y judío (minoría sobrerrepresentada a nivel universitario), reclamó no haber podido ingresar a la Facultad de Derecho de la Universidad de Washington por los puntos extras que dicha escuela otorgaba a ciertas minorías, de manera que de haber sido negro, filipino, chicano o indio americano hubiese sido admitido. DeFunis solicitó se declarara que el método de selección de la Universidad era inconstitucional porque violaba su derecho a la igualdad protegida por la Decimocuarta Enmienda, ya que utilizaba el origen racial como estándar para la clasificación de los candidatos. La Suprema Corte del estado de Washington falló contra el demandante pues consideró que el método de selección de la Universidad no violaba la igualdad, pues el origen racial era solo uno de los elementos que los comités de evaluación consideraban relevantes para la admisión definitiva. Si bien, finalmente, el caso no fue resuelto por la corte federal (pues De Funis fue admitido provisionalmente), Dworkin apoyó la decisión del tribunal de Washington argumentando que la cláusula de igual protección ante la ley no contiene ninguna concepción particular de la igualdad, "de manera que no es razonable sostener que todas las distinciones raciales son intrínsecamente lesivas para el derecho contenido en la Decimocuarta Enmienda" (2003:452).

Otro caso importante fue resuelto en 1995 por el Tribunal de Justicia de las Comunidades Europeas de Luxemburgo con ocasión de la obtención de un puesto de trabajo, en Bremen, por aplicación de una norma que establecía preferencia en la contratación de mujeres frente a candidatos masculinos con la misma capacitación si, en el sector de que se trataba, había subrepresentación. Kalanke, al perder el puesto frente a una mujer, demanda que la norma se declare contraria a la Constitución alemana. El Tribunal de Justicia de la Comunidad Europea resuelve que la normativa interna contradecía la Directiva Comunitaria que garantiza la igualdad, pues establecía -en una opinión muy discutida- "prioridad absoluta e incondicionada a la mujer en caso de nombramiento o promoción” ${ }^{4}$. Atienza critica la resolución sobre la base de que una medida de discriminación inversa es legítima si a) es razonablemente eficaz para alcanzar un objetivo; b) persigue un objetivo socialmente importante y; c) no haya modo alternativo para alcanzar dicho objetivo, por lo que se trataba de una norma de discriminación inversa válida (1996:119-22). Al modificarse la cuestionada Directiva en septiembre de 2002,

${ }^{4}$ Para un análisis completo del caso, véase Ruiz Miguel, Alfonso (1996): "La discriminación inversa y el caso Kalanke", en Revista Doxa N 19. 
las diferencias de trato basadas en características relacionadas al sexo han dejado de verse como excepciones al principio de igualdad de trato, en la medida que cumplan con los requisitos allí establecidos. Del mismo modo, la igualdad de oportunidades se entiende comprendida en su versión de "igualdad de resultados" y la igualdad sustantiva cobra protagonismo, relegando a la tradicional igualdad formal ${ }^{5}$ (Salazar, 2004:10).

Noelle Lenoir ha planteado que la paridad obligada es una medida comparable con el derecho al voto y la separación de poderes, pues reflejan que el espontáneo desarrollo de la práctica política no basta para asegurar el progreso de la situación política de las mujeres (Irving, 2008:121). Francia, al igual que Italia, precisó de una enmienda constitucional para provocar el cambio, y fue el propio Consejo Constitucional quien esbozó esta necesidad en 1999, al rechazar un nuevo estatuto que establecía la paridad entre hombres y mujeres, por ser contrarias a la Constitución. Así, en 1999 Francia aprobó una enmienda constitucional que estableció el principio de la paridad ${ }^{6}$ para luego, en junio del 2000, promulgar la ley de igual acceso de hombres y mujeres a cargos electorales y funciones, introduciendo la paridad en la representación política.

Por su parte, la Corte Constitucional española ha entendido la prohibición constitucional de discriminar a base del sexo desde una perspectiva sustantiva del principio de igualdad, por tanto, las acciones afirmativas que se adoptan en el marco de esta norma constitucional no solo son acordes, sino que además "son legitimadoras de la igualdad sustantiva” (Rubio-Marín, 2005:259). En esa línea va la ley orgánica 3/2007 sobre igualdad efectiva de mujeres y hombres que dispuso que en las votaciones electorales deben incluirse no menos del $40 \%$ de candidatos de cada género (Rodríguez-Ruiz y Rubio-Marín, 2012:194).

En Colombia, gracias a la influencia de la CEDAW, se estableció a nivel constitucional "la prohibición de discriminación contra las mujeres y la autorización de medidas positivas especiales como medios para asegurar la igualdad sustantiva, más que simplemente la formal" (Morgan, 2005:78). Así, en 1991 la Corte Constitucional Colombiana consideró que la discriminación a favor de las hijas de cierta clase de uniformados por sobre los hijos (en el otorgamiento de ciertos beneficios económicos) era una medida legítima tendiente a hacer real y efectivo el principio de igualdad, "dada la dependencia económica frecuente de las mujeres sobre los hombres en la sociedad colombiana" (Morgan, 2005:87). Finalmente, en 1999 se estableció a nivel constitucional que "las autoridades garantizarán la adecuada y efectiva participación de la mujer en los niveles decisorios de la Administración Pública”, permitiendo la aprobación de una Ley de

\footnotetext{
${ }^{5}$ Los Estados miembros podrán disponer, por lo que respecta al acceso al empleo, incluida la formación pertinente, que una diferencia de trato basada en una característica relacionada con el sexo no constituye discriminación cuando, debido a la naturaleza de las actividades profesionales concretas o al contexto en que se lleven a cabo, dicha característica constituya un requisito esencial y determinante, siempre y cuando el objetivo sea legítimo y el requisito proporcionado" (Apartado 6, art. 2, Directiva N 76/207).

${ }^{6}$ Esta enmienda añadió al art. 3 el siguiente texto: "los estatutos deben promover el igual acceso de mujeres y hombres a oficinas y posiciones electorales", y al artículo 4 una disposición final que establece que "los partidos políticos deben contribuir en la implementación del principio [de paridad]".
} 
Cuotas que sigue la idea de "masa crítica" de Naciones Unidas y el porcentaje aceptado por Colombia en la Plataforma de Beijín 95, de 30\%

Por último, la Corte Suprema chilena ha establecido que el principio de igualdad contenido en la Constitución no puede entenderse en forma rígida y dogmática, por lo que la ley puede hacer diferenciaciones entre personas o grupos que no impliquen discriminaciones arbitrarias o supongan diferencias o privilegios que deterioren o destruyen esa igualdad. Y concluye: "la discriminación positiva establece que las normas impugnadas no son inconstitucionales, porque su objetivo es (...) la protección de cierta categoría de personas para resguardar su debilidad social, cultural y económica frente a otros estamentos de la sociedad chilena que no se encuentran en las mismas condiciones, como lo son los indígenas del país" (Figueroa, 2014:17).

El impulso decisivo en materia de cuotas vino desde Beijing 95, donde se aunaron voluntades y esfuerzos enfocados directamente a concientizar a los Estados de la necesidad de adoptar en el corto plazo medidas de discriminación positiva como los sistemas de cuotas electorales. Se observó que, a nivel mundial, entre 1930 y 1980 solo 10 países contaban con un sistema de cuotas, a los que se adicionaron 12 en la siguiente década hasta alcanzar más de 50 Estados en la década del 90 y otros 40 desde el 2000. A nivel regional las cuotas se impulsaron en 11 Estados y entre 1996 y 1997 nueve de los 11 países latinoamericanos con cuotas las incorporaron a su legislación. Así, hoy, más de 100 países en el mundo cuentan con política de cuotas, lo que parece "reflejar una norma internacional creciente respecto de la necesidad de promover la representación política de las mujeres" (Lena Krook, 2008:29).

\subsection{Modalidades de cuotas de género electorales}

Las cuotas de género admiten tres modalidades: los escaños reservados, las cuotas de partidos y las cuotas legislativas. Los escaños reservados guardan un puesto a las mujeres en las asambleas políticas que es, generalmente, entre el 1 y el $2 \%$ de todos los escaños, a excepción de lo sucedido en Tanzania donde se implementó esta modalidad con una exigencia del $30 \%$ de la presencia femenina. Este tipo de cuotas son propias de África, Asia y Medio Oriente, todos territorios en que la representación femenina es muy baja y se alcanza solo con reformas constitucionales. A su turno, las cuotas de partido son mecanismos voluntarios que motivan una proporción determinada de mujeres entre los candidatos al Parlamento. La proporción que exigen es más alta que la de los escaños reservados, oscilando entre 25 y $50 \%$ y permite incluir a la vida partidista a quienes,

${ }^{7}$ Si bien la Corte Constitucional reconoce que "las acciones positivas, incluyendo la discriminación inversa, están expresamente autorizadas en la Constitución, la que garantiza no solo el principio de no discriminación, sino también alude a la dimensión sustantiva de la igualdad que tiene un carácter remedial, compensatorio, emancipatorio, correctivo y defensivo de personas y grupos situados en condiciones de inferioridad", ha descartado la posibilidad de incorporar cuotas electorales obligatorias sobre la base de que la autonomía de los partidos en el campo electoral debe prevalecer (Rodríguez-Ruiz y Rubio-Marín, 2012:192). 
en otras circunstancias, quedarían afuera bajo el argumento de "carencia natural" de talento (Héritier, 2007:230).

Finalmente, las cuotas legislativas constituyen medidas decretadas por los Parlamentos nacionales que vinculan a todos los partidos políticos y que los obligan a nominar una cierta proporción de candidatas femeninas. Se asemejan a las cuotas de partidos, pues tienden a interferir en el proceso de selección más que en los resultados de las elecciones. Propias de países en vías de desarrollo, como los que habitan Latinoamérica, las cuotas legislativas requieren de una presencia femenina que va entre el 25 y el $50 \%$ (Lena Krook, 2008:32). Se implementan mediante reformas a las Constituciones o a las leyes electorales buscando legitimar la acción afirmativa y reconocer el "género" como una entidad política.

Ahora, numerosos estudios han señalado que el sistema electoral es relevante al momento de implementar con éxito un régimen de cuotas de género. Se sostiene que el sistema que más favorece las cuotas es el de representación proporcional, ya que en él los partidos tienen un incentivo para "equilibrar" sus listas incluyendo aspirantes que tengan nexos con una gama variada de grupos sociales y de postular candidatos o candidatas que representen diferentes facciones y sectores del electorado. En cambio, en los sistemas de representación mayoritaria, donde solamente hay un candidato por circunscripción, se genera una presión que, sumado a los prejuicios, resulta en la nominación mayoritaria de hombres. A su turno, si bien hay quienes prefieren las listas cerradas (como Peschard) otros consideran que un sistema de lista abierta es igualmente positivo, pues permite que el votante elija a una mujer "independientemente de la posición en que sean colocadas por los dirigentes dentro de la lista” (Htun, 2002:31). Por último, son preferibles los distritos de grandes magnitudes, pues son mayores las oportunidades para que estas encuentren un lugar en la representación por ser más alto el número de candidatos/as.

Otro de los factores que favorecen el éxito en la implementación de las cuotas es fijar una ubicación específica para las candidaturas femeninas, además de que el cumplimiento de la regla sea requisito indispensable para el registro de las listas de candidatos de cada partido. Asimismo, debiera velarse por una redacción de las leyes de cuotas que evite la ambigüedad y vaguedad en su implementación, pues se ha visto que, cuando hay lagunas, los partidos dan por cumplida la cuota colocando a las mujeres en puestos suplementarios o decorativos en las listas. Así ocurrió, por lo demás, en Brasil, donde se consideró que la norma que obligaba a los partidos a reservar el $30 \%$ de los puestos de las listas a aspirantes mujeres no les exigía realmente "llenar" esos puestos (Htun, 2002:36). Luego, es preciso que las cuotas tengan asociadas sanciones a su incumplimiento, las que deben ser aplicadas por entidades supervisoras independientes. En el caso de Francia, la reforma constitucional del 2000 obligó a los partidos políticos a nominar $50 \%$ de hombres y $50 \%$ de mujeres candidatas bajo sanciones financieras y políticas.

Un ejemplo de éxito de las cuotas es Argentina, pionero en introducirlas en 1991 y que para el 2002 alcanzó 30,7\% de participación femenina en la Cámara Baja y 35\% en la Cámara Alta. Costa Rica también fue exitosa en su implementación, mediante una ley en 1997 que obligó a los partidos políticos a reservar un mínimo de $40 \%$ de las candidaturas a mujeres. Aunque después de la primera elección solo se aumentó la 
participación femenina en $16 \%$, el resultado fue menor que el requerido por la cuota, por lo que en el 2002 el Tribunal Supremo Electoral ordenó a los partidos políticos situar a las mujeres en puestos "elegibles" dentro de sus listas de candidatos, provocando un incremento importante en el número de mujeres congresistas, alcanzando el primer lugar a nivel mundial en cuanto a representación de mujeres en el órgano legislativo (Olsen, 2002:148-149).

Leyes de cuota y elección de mujeres en América Latina*

\begin{tabular}{|c|c|c|c|c|c|c|}
\hline País & $\begin{array}{l}\text { Año de } \\
\text { la ley }\end{array}$ & $\begin{array}{c}\% \\
\text { cuota }\end{array}$ & $\begin{array}{c}\text { Rama } \\
\text { legislativa }\end{array}$ & $\begin{array}{l}\% \text { de mujeres } \\
\text { antes de la ley }\end{array}$ & $\begin{array}{l}\% \text { de mujeres } \\
\text { después de la ley }\end{array}$ & $\begin{array}{c}\text { Cambio } \\
\text { en } \%\end{array}$ \\
\hline Argentina & 1991 & 30 & Cámara baja & 6 & 28 & 22 \\
\hline \multirow{2}{*}{ Bolivia } & 1997 & 30 & Cámara baja & 11 & 12 & 1 \\
\hline & 1997 & 25 & Senado & 4 & 4 & 0 \\
\hline Brasil & 1997 & 25 & Cámara baja & 7 & 6 & -1 \\
\hline Costa Rica & 1996 & 40 & Unicameral & 14 & 19 & 5 \\
\hline República Dominicana & 1997 & 25 & Cámara baja & 12 & 16 & 4 \\
\hline Ecuador & 1997 & 20 & Unicameral & 4 & 17 & 13 \\
\hline \multirow{2}{*}{ México* } & 1996 & 30 & Cámara baja & 14 & 17 & 3 \\
\hline & 1996 & 30 & Senado & 13 & 15 & 2 \\
\hline Panamá & 1997 & 30 & Unicameral & 8 & 11 & 3 \\
\hline \multirow{2}{*}{ Paraguay } & 1996 & 20 & Cámara baja & 3 & 3 & 0 \\
\hline & 1996 & 20 & Unicameral & 11 & 20 & 9 \\
\hline Perú & 1997 & 30 & Unicameral & 11 & 18 & 7 \\
\hline \multirow{2}{*}{ Venezuela } & 1998 & 30 & Cámara baja & 6 & 13 & 7 \\
\hline & 1998 & 30 & Senado & 8 & 9 & 1 \\
\hline Promedio & & 28 & & 9 & 14 & 5 \\
\hline
\end{tabular}

Con reelaboración de Cecilia Lavalle Torres: "Derechos políticos de las mujenes y cuotas de género. Ruta cuesta arriba”, Foro virtual CIMAC, 1 junio 2012.

Otro caso es el de los países escandinavos, estos no han necesitado de la implementación de cuotas a nivel constitucional o legal para alcanzar una elevada representación femenina en el ámbito político, equivalente a $40 \%$ del total de los parlamentarios. Aquí cobraron relevancia las presiones de grupos de mujeres ejercidas directamente sobre los partidos políticos y sus aparatos internos, así como las del movimiento de mujeres 
en general. Esta presión se tradujo en la implementación de cuotas a nivel de partido, como fue el caso de los socialdemócratas y de izquierda que introdujeron el sistema de cuotas durante la década del 70 y del 80, no obstante que "la mayor parte de los partidos de centro y de derecha consideraron a este mecanismo como no liberal" (Dahlerup, 2002:167). Finalmente, veamos qué ha ocurrido en el resto del mundo.

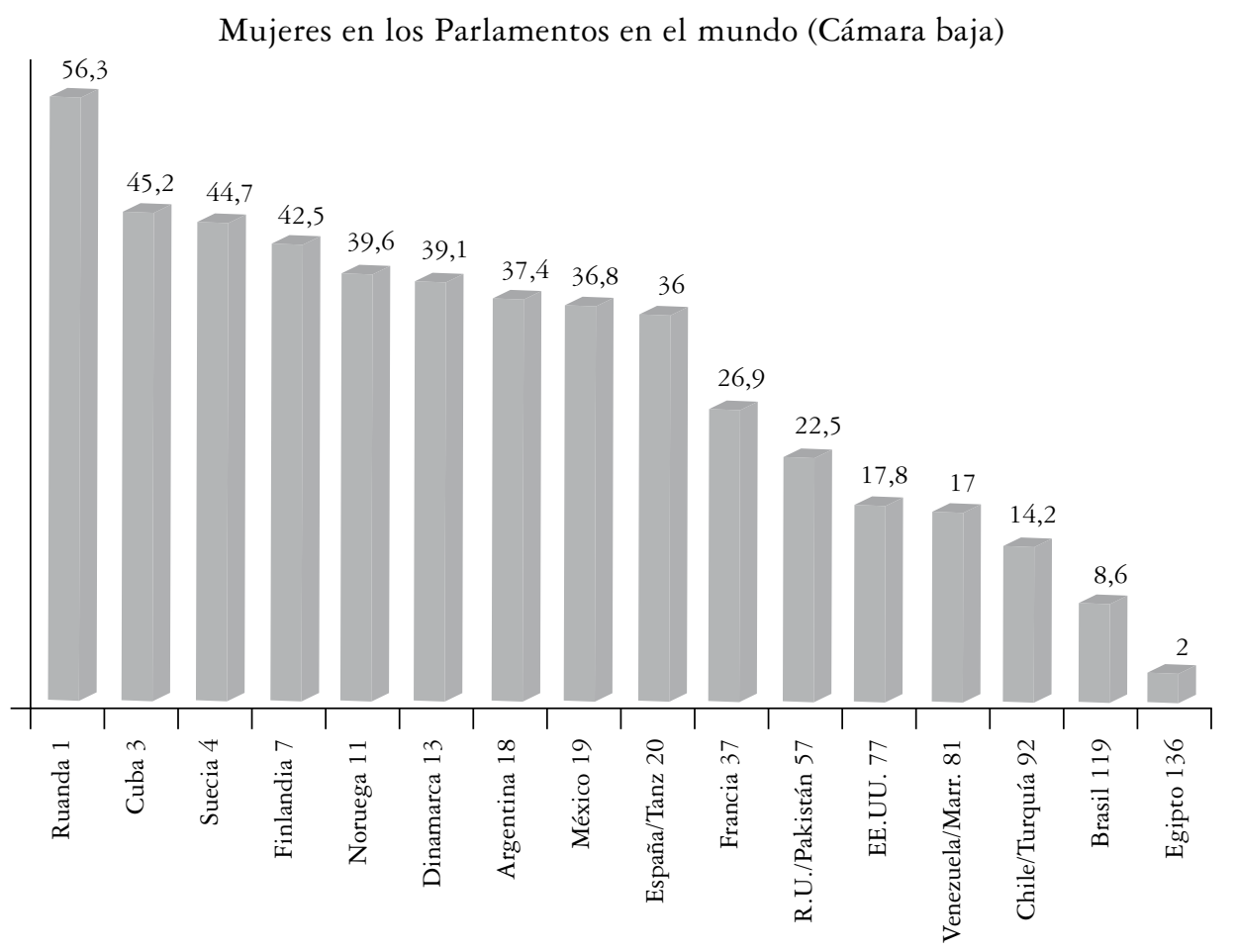

Fuente: Unión Interparlamentaria (actualizada 24-4-2013).

\subsection{El mérito y las cuotas electorales}

Las medidas de discriminación positiva poseen dos particularidades que las convierten en una paradoja en sí mismas. Por una parte está el hecho de que la discriminación inversa o positiva utiliza los mismos rasgos que se denuncian en la discriminación injusta, como son "el sexo, la raza o similares rasgos que tienen la cualidad de ser transparentes e inmodificables para los individuos que las portan y son considerados por la sociedad tendencial y generalmente de forma negativa" (Zúñiga et al., 2007:15). En segundo lugar, se aplican a la distribución de recursos escasos, lo que provoca cierto conflicto social. Además, la carga emotiva de la expresión "discriminación” perjudica su significado cognoscitivo, debido a que si una palabra funciona como una condecoración o 
como un estigma, la gente va manipulando arbitrariamente su significado para aplicarlo a los fenómenos que acepta o repudia, sin mayor análisis. Luego, lo importante es ser cuidadoso de no caer en la tentación inmediata de resolver verbalmente el problema de su justificación (Ruiz Miguel, 1996:127).

De otra parte, se argumenta que el mecanismo de las cuotas es contrario al principio de igualdad, pues este proscribiría toda diferencia basada en criterios no relevantes, como son la raza o el sexo. El problema de este argumento es que olvida que el tratamiento diferenciado es una de las modalidades del principio de igualdad, por lo que frente a ciertas situaciones el mandato que dirige el principio es precisamente diferenciar en el trato. Luego, para tratar a las personas con igual consideración y respeto se ordena la aplicación de medidas que impliquen el trato diferenciado, cuya desventaja aparejada se ve compensada con el beneficio social que este reporta.

Hay quienes arguyen que las cuotas harían a las mujeres víctimas de una nueva estigmatización social porque se les asignarían puestos con prescindencia del criterio del mérito, de modo que unos serán elegidos por su competencia mientras que otras por causa de las cuotas y bajo sospecha de deber su promoción profesional o política no tanto a sus capacidades sino a su sexo. Dworkin argumenta que ningún hombre puede reclamar que sus derechos son infringidos por una ley de cuotas, ya que no es posible argumentar que merezca a priori (debido a sus mayores "méritos") acceder a un cargo. Por ello, la idea de mérito en abstracto es equívoca, debido a que este no existe sin referencias a ciertos objetivos institucionales previamente definidos como valiosos [y] será cada institución la que decida qué es, para el caso concreto, lo que se necesita para acceder al cargo (2003:451).

Lo cierto es que estas objeciones defienden la meritocracia bajo el supuesto de que las cuotas y otras medidas de acción positiva no dejan que ganen los mejores. Pero ello supone creer que ahora solo están los mejores en el Parlamento, cuestión que es difícil de defender. Los partidos tienden a nominar a sus candidatos a base de cálculos relacionados con sus redes familiares y sociales, sus años de militancia y el sexo. No se elige "a los mejores” en el sentido común en el que usualmente se entiende el término (quien tiene el mejor currículo y experiencia), pues se aplica la llamada "regla del reconocimiento endógeno", por la que en los nombramientos individuales, incluidos los cargos políticos, se reconoce mérito inmediato a la persona que refleje a aquellos que ya están en el poder" (Irving, 2008:131). Los cuales son, casi todos, hombres.

El discurso público sobre las cuotas tergiversa nuestro sistema actual, pues asumen que ellas socavan uno de nuestros valores sociales más importantes: el mérito. Pero lo que ocurre realmente es que se hace valer un sesgo inconsciente claramente demostrado en los estudios con los que comenzamos este trabajo. Que las mujeres son familiares y cálidas, adecuadas para las tareas de cuidados tanto de niños como de ancianos y enfermos. En cambio, los hombres, en tanto agentes, son naturalmente aptos para la autoridad y el liderazgo. Se trata de una respuesta diseñada para proteger el statu quo al representar falsamente el impacto de las cuotas como contrarias a la idea de mérito.

Es un mito que las cuotas socavan el mérito. Las cuotas son en realidad una forma de convertir nuestros actuales sistemas de ascenso y promoción defectuosos, en una 
verdadera meritocracia. En virtud de que la sociedad, según demuestran los estudios, construye sus instituciones basada en prejuicios y estereotipos solapados contra grupos excluidos, asignándoles capacidades inferiores, se precisa de la presencia de mecanismos de acción positiva que logren mitigar la influencia de esos prejuicios y la ceguera de las instituciones y las personas que toman decisiones. El mérito-como concepto socialmente construido- no solo valora talentos, sino que también le da valor a la pertenencia a un determinado sexo, pues se cree que no sería lo mismo elegir a John o Howard que a Jennifer o Heidi.

\section{CONCLUSIÓN}

Quienes sostienen la importancia del género afirman que hacer visibles a los grupos históricamente marginados (como las mujeres) mediante la ocupación de cargos de elección popular interpela los estereotipos arraigados respecto de que ciertos grupos no son adecuados para ostentar cargos políticos, a la vez que aumentan la legitimidad de facto del sistema de gobierno en contextos de discriminación en el pasado. Por otra parte, se sostiene que es preferible que los representantes sean descriptivamente similares a sus representados, ya que al compartir experiencias comunes serían más propensos a actuar a favor del grupo social del cual provienen, promoviendo sus intereses. Asimismo, algunos estudios afirman que el número de mujeres representantes políticas sí importa, pues ayuda a conformar una masa crítica desde el espectro político mismo que, aun sin exigirse en clave paritaria, es capaz de cumplir una función de "denuncia y de estímulo para la superación de la desigualdad general de las mujeres” (Ruiz Miguel, 2001:255).

La experiencia vivida en Latinoamérica da fuerza a la idea de que el género es importante, pues el aumento de parlamentarias coincidió con un interés en los derechos de las mujeres relativos a la violencia doméstica y los derechos reproductivos sin precedentes. Durante los años 90 dieciséis países aprobaron leyes para proteger a las víctimas de la violencia doméstica y se lanzaron programas para mejorar la salud reproductiva. Junto con ello, varios países reformaron sus Constituciones para proteger la igualdad de los derechos de ambos sexos, entre otras modificaciones legales relativas al Derecho de Familia. Ello no significa olvidar la heterogeneidad de las mujeres basándose en ideas esencialistas sobre cuáles son sus intereses como grupo y atribuyéndoles "competencias naturalmente femeninas (...) una sensibilidad específica de la mirada femenina” (Héritier, 2007:233). Nada de eso, la defensa de las cuotas se estructura en términos de pluralismo, derechos iguales, ampliación de la representación y mejora de los procesos de democratización.

Las mujeres transitan por todo tipo de factores, como el origen étnico, la clase social, la religión, la política, orientación sexual, etc. De modo que su definición es tan amplia como tantas pueden llegar a ser las definiciones contenidas en cada una de estas categorías. La política de la presencia, rehuyendo de identidades esencialistas y de representaciones de intereses exclusivos, defiende que la dualidad humana de por sí es razón suficiente para cuestionar el monopolio de los varones en el ámbito de la política. La discrepancia sistemática entre la participación de las mujeres en los puestos intermedios e inferiores 
de las organizaciones y su presencia en el proceso de toma de decisiones es una evidencia patente de una injusticia, por lo que el discurso que, en clave democrática, defiende que hombres y mujeres son capaces de representar al otro indistintamente, choca con la realidad de que "en la práctica, solo los hombres parecen calificados para representar a ambos sexos" (Héritier, 2007:232).

La población calificada para ocupar cargos de alta responsabilidad política ha sido igualitariamente distribuida entre hombres y mujeres, y el balance se está inclinado todo el tiempo a favor de estas últimas. Por tanto, debido a la escasa participación de las mujeres en estos cargos, solo se puede llegar a una conclusión: "Si a pesar de la existencia hoy de la igualdad en el punto de partida, el punto de llegada sigue siendo desigual, esto es, porque no es el mérito, o no solo este, el que determina que la mayoría de las más altas responsabilidades del Estado estén en manos de los hombres" (Morgan, 2005:89).

Las cuotas colaboran para erradicar las desigualdades que relegan derechamente a las Jennifer(s) a la esfera íntima doméstica o, en casos más felices, a espacios de la esfera pública que replican los ámbitos "inferiores" de la privada, y que siguen posicionando a los John(s) en los espacios públicos privilegiados, con proyección de mejores salarios y mayor estatus social. Por ello, no debemos cesar en el esfuerzo de implementar mecanismos de compensación social que permitan superar los estereotipos y permitir a las mujeres el acceso a la cuota de poder que les corresponde.

\section{BIBLIOGRAFÍA}

Alexy, Robert (2008). Teoría de los derechos fundamentales, 2a. Edición, Centro de Estudios Políticos y Constitucionales, Madrid.

Archenti, Nélida (2002). "Los caminos de la inclusión política, acciones afirmativas de género", en Hombres públicos, mujeres públicas, Fundación Friedrich Ebert, Buenos Aires.

AtienZa, Manuel (1996). "Un comentario al caso Kalanke", Doxa No 19.

Ballington, Julie y Méndez-Montalvo, Myriam (2002). "Introducción”, en Mujeres en el Parlamento. Más allá de los Números. International IDEA, Estocolmo.

Beltrán, Elena (2001). "Justicia, democracia y ciudadanía: las vías hacia la igualdad”, en Feminismos. Debates teóricos contemporáneos, Elena Beltrán y Virginia Maquieira (eds.), Alianza Editorial, Madrid.

Buvinic, Mayra y Roza Vivian (2004). La mujer, la política y el futuro democrático de América Latina, BID, Serie de Informes Técnicos, WID-108, Washington DC.

Dahlerup, Drude (2002). "El uso de cuotas para incrementar la representación política de la mujer", en Mujeres en el Parlamento. Más allá de los Números. International IDEA, Estocolmo.

Dworkin, Ronald (1984). Los derechos en serio, Ariel, Barcelona (2003). Virtud Soberana. La Teoría y la Práctica de la Igualdad, Paidós, Barcelona.

FACIO, Alda, Jimenez, Rodrigo y Morgan, Martha (2005). "Gender Equality and International Human Rights in Costa Rican Constitucional Jurisprudence". The Gender of Constitutional Jurisprudence, Beverly Baines y Ruth Rubio-Marín (Eds), Cambridge University Press, New York.

Franceschet, Susan (2008). “¿Promueven las cuotas de género los intereses de las mujeres? El impacto de las cuotas en la representación sustantiva de las mujeres”, en Mujer y política. El impacto de las cuotas de género en América Latina, Marcela Ríos (ed.) IDEA, FLACSO-Chile, Santiago. 
Figueroa, Rodolfo (2014). “ ¿Son constitucionales las cuotas de género para el Parlamento?”. Artículo en arbitraje.

Hérittier, Françoise (2007). Masculino/Femenino II. Disolver la jerarquía, Fondo de Cultura Económica de Argentina, S.A, Buenos Aires.

Hirschl, Ran y Shachar, Ayalet (2005). "Constitutional Transformation, Gender Equality, and Religious/National Conflict in Israel: Tentative Progress through the Obstacle Course”. The Gender of Constitutional Jurisprudence, Beverly Baines y Ruth Rubio-Marín (Eds), Cambridge University Press, New York.

Htun, Mala (2002). "Mujeres y poder político en Latinoamérica”, en Mujeres en el Parlamento. Más allá de los Números, International IDEA, Estocolmo.

LAPORTA, Francisco (1985). "El principio de igualdad. Introducción a su análisis", en Sistema (Revista de Ciencias Sociales) $\mathrm{N}^{\circ}$ 67, Madrid.

IRving, Helen (2008). Gender and the Constitution. Equity and Agency in comparative constitutional design, Cambridge University Press, Nueva York.

Lena Krook, Mona (2008). "La adopción e impacto de las leyes de cuotas de género: una perspectiva global", en Mujer y política. El impacto de las cuotas de género en América Latina, Marcela Ríos (ed.) IDEA, FLACSO-Chile, Santiago.

Leuchprecht, P (1996). "El acceso de las mujeres a los procesos de adopción de decisiones políticas”, en Mariño Menéndez, F. (Ed.). La protección internacional de los derechos de la mujer tras la Conferencia de Pekín, Universidad Carlos III de Madrid-BOE, Madrid.

Mansbridge, Jane (1999). "Should Blacks Represent Blacks and Women Represent Women? A Contingent 'Yes'”. Journal of Politics 61, 3: 628-657.

Millard, Eric (2005). "Constituting Women: The French Way". The Gender of Constitutional Jurisprudence, Beverly Baines y Ruth Rubio-Marín (Eds), Cambridge University Press, New York.

Morgan, Martha (2005). "Emancipatory Equality: Gender Jurisprudence under the Colombian Constitution". The Gender of Constitutional Jurisprudence Beverly Baines y Ruth Rubio-Marín (Eds.), Cambridge University Press, New York.

Moss-RaCusin et al. "Science faculty's subtle gender biases favor male students" (2012). Proceedings of the National Academy of Sciences of the United States of America (PNAS), October, vol. $109 \mathrm{~N}^{\circ} 41$.

OlsEn, Karen (2002). "El camino hacia la igualdad. Las mujeres costarricenses en el Parlamento", en Mujeres en el Parlamento: más allá de los números, Myriam Méndez-Montalvo y JulieBallington (eds.), IDEA, Estocolmo.

Peschard, Jacqueline (2002). "El sistema de cuotas en América Latina. Panorama general”, en Mujeres en el Parlamento: más allá de los números, Myriam Méndez-Montalvo y Julie Ballington (eds.), IDEA, Estocolmo.

Phillips, Anne (1995). The Politics of Presence. Clarendon Press, Oxford.

Puyol, Ángel (2012). El sueño de la igualdad de oportunidades. Crítica a la ideología del mérito, Gedisa, Barcelona.

Rawls, John (1971). Theory of justice, Cambridge University Press. (2002). La justicia como equidad, Paidós, Barcelona.

Ríos, Marcela (2006). Cuotas de género: democracia y representación. FLACSO-Chile, Santiago. (2008). "Introducción", en Mujer y política. El impacto de las cuotas de género en América Latina, Marcela Ríos (ed.), IDEA, FLACSO-Chile, Santiago.

Rodríguez, Jesús (2009). "El principio rawlsiano de diferencia: dilemas de interpretación". Enrabonar: Quaderns de filosofía, $\mathrm{N}^{\circ} 43$. 
Rodríguez, Blanca y Sacksofsky, Ute (2005). “Genderin the German Constitution”, en The Gender of Constitutional Jurisprudence, Beverly Baines y Ruth Rubio-Marín (Eds), Cambridge University Press, New York.

Rodríguez, Blanca y Rubio-Marín, Ruth (2012). “On Parity, Interdependence, and Women's Democracy”. Feminist Constitutionalism. Global Perspectives, Beverley Baines, Daphne BarakErez y TsviKahana (Eds), Cambridge University Press, New York.

Rubio-Marín, Ruth (2005). "Engendering the Constitution: The Spanish Experience”. The Gender of Constitutional Jurisprudence Beverly Baines y Ruth Rubio-Marín (Eds), Cambridge University Press, New York.

Salazar, Octavio (2004). "Las Mujeres y la Constitución Europea. La insoportable 'levedad' del género en la Unión Europea". Disponible en: http://helvia.uco.es/xmlui/bitstream/ handle/10396/2240/FCIAOSB.pdf? sequence $=1$

Ruiz, Miguel (2001). "La representación democrática de las mujeres”, en Anales de la Cátedra de Francisco Suárez $\mathrm{N}^{\circ}$ 35, Universidad de Granada.

Singer, Peter (1995). Ética Práctica, $2^{\circ}$ Edición, Cambridge University Press, New York.

ToEgel and Barsoux. "Women Leaders: The Gender Trap", The European Business Review, Número de julio de 2012.

Wood, Huangb, Wood y Murray (2014) "Investors prefer entrepreneurial ventures pitched by attractive men". Proceedings of the National Academy of Sciences. vol. $111 \mathrm{~N}^{\circ} 12$ : 4427-4431.

ZúÑIga Añazco, Yanira (2005). "Democracia paritaria: de la teoría a la práctica”, en Revista de Derecho, Vol. XVIII - No 2, Valdivia, Chile: 131-154.

ZúÑIga Fajuri, Alejandra (2013). "Justicia y genética: compensando las diferencias", Ideas y valores, Vol. 62, No 151.

ZúÑIgA, Alejandra; Aguilera, Daniela y Vásquez, Andrea (2007). "Lejos del Poder. Hacia la implementación de una Ley de Cuotas en Chile”, en Revista de Derecho, Vol. XX - No 2. 